GRASAS Y ACEITES, 57 (3),

JULIO-SEPTIEMBRE, 319-327, 2006,

ISSN: 0017-3495

\title{
Amphoteric surfactants containing $\alpha$-hydroxy ester group and an amino acid residue
}

\author{
By A. M. F. Eissa \\ Chemistry Department, Faculty of Science, Benha University, Benha - Egypt. \\ E-mail: ref_at@hotmail.com
}

\section{RESUMEN}

Tensioactivos anfóteros conteniendo un grupo alfa hidroxi éster y un residuo de aminoácido

Se prepararon una serie de tensioactivos anfóteros conteniendo un grupo alfa hidroxi éster y un residuo de aminoácido por adición de derivados epoxy (obtenidos mediante epoxidación de metacrilato de alquilo) a diferentes tipos de aminoácidos (glicina, alanina, valina, isoleucina, fenilalanina tirosina, serina, treonina y ácidos aspártico y antranílico). Las estructuras de los compuestos preparados se confirmaron por los espectros de infrarrojo, de masa, resonancia magnética nuclear de protones y análisis elemental. Se determinaron la tensión superficial, el punto de Kraft, el poder espumante, la concentración micelar crítica en emulsión y las estabilidades de $\mathrm{Ca}++$. También se estudiaron la actividad antimicrobiana y la biodegradabilidad.

PALABRAS-CLAVE: Actividad antimicrobiana - Biodegradabilidad - Tensioactivos anfóteros.

\section{SUMMARY}

Amphoteric surfactants containing $\alpha$-hydroxy ester group and an amino acid residue

A series of amphoteric surfactants containing $\alpha$-hydroxy ester group and an amino acid residue were prepared with the addition of epoxy derivatives (which were prepared from epoxidation of alkyl methacrylate) to different types of amino acids (glycine, alanine, valine, isoleucine, phenylalanine, tyrosine, serine, threonine, aspartic and anthranilic acid). The structures of the prepared compounds were confirmed by infrared spectra, proton magnetic resonance spectra, Mass spectra and elementary analysis. Surface tension, Kraft point, foaming power, critical micelle concentration emulsion and $\mathrm{Ca}^{++}$stabilities were determined. Antimicrobial activity and biodegradability were also screened.

KEY-WORDS: Anphoteric surfactants - Antimicrobial activity - Biodegradability.

\section{INTRODUCTION}

As a part of our program (Eissa., 2002; Eissa et al. 1996; Eissa. 1995; Eissa et al. 2003; Amin et al., 2004; El-Dougdoug et al., 2001) on the synthesis and characterization of different types of surface active agents, the author attempts to synthesize a novel group of amphoteric surfactants based on amino acid residue. Amino acids are not only essential components of the human body but also interesting raw materials for surfactants (Nasreddine et al., 1993). The presence of anionic and cationic moieties in the molecule of an amino acid makes it possible to prepare various kinds of surfactants by introducing a hydrophobic group into the molecule (KIrvens., 1953; Herrmann., 1963; Tokiwa et al., 1967; Bluestein et al., 1973; Andersen., 1957).

Sodium salts of long chain $\mathrm{N}$-alkyl- $\beta$-alanines are well known amphoteric surfactants. Surfactants of the $\beta$-alanine type have been used in such applications as shampoos, cosmetics, emulsion paints, various products in the textile industry, corrosion inhibitors, industrial cleaning products and many others (Hikota., 1979). One of the most important features of these surfactants is that they are effective over a wide $\mathrm{pH}$ range, in their cationic form in an acidic solution, and in their anionic form in an alkaline solution, except at the isoelectric points. Furthermore, these surfactants are less toxic to higher animals and are not irritating to human skin (Christophe et al., 2002; Marion et al., 2002; Hironari et al., 2002; Infante et al., 2003).

The present paper deals with the preparation and functional properties of a series of amphoteric surfactants containing the $\alpha$-hydroxy group and an amino acid residue. A series of these compounds was synthesized from alkyl methacrylate as raw starting material (alkyl; a, $\mathrm{C}_{8} \mathrm{H}_{17} ; \mathrm{b}, \mathrm{C}_{12} \mathrm{H}_{25} ; \mathrm{c}, \mathrm{C}_{18} \mathrm{H}_{37}$ ).

\section{MATERIAL AND METHODS}

The IR spectra in $\mathrm{KBr}$ were recorded on a Shimadzu 470 Spectrometer. The ${ }^{1} \mathrm{H}$ NMR were measured on Varian EM-390-90 MHz a spectrometer using TMS as internal reference and the chemical shifts are expressed as $\delta$ (ppm). The mass spectra were recorded on HP Model: MS 5988 at $70 \mathrm{eV}$. The physical and spectral data are listed in (Tables 1 and 2).

\subsection{General procedure of formation of epoxy fatty ester (2a-c)}

Alkyl methacrylate 1a-c (1 mmole) (Figure 1) in glacial acetic acid $(40 \mathrm{ml})$ was mixed with $8 \%$ aqueous sodium hydroxide $(12 \mathrm{ml})$ followed by the addition of hydrogen peroxide $(30 \% 5 \mathrm{ml})$. The 
Table 1.

Physical data of the synthesized compounds

\begin{tabular}{|c|c|c|c|c|c|c|c|}
\hline \multirow[b]{2}{*}{ Compds } & \multirow[b]{2}{*}{$\mathbf{R}$} & \multirow[b]{2}{*}{$\mathbf{R}^{\prime}$} & \multirow[b]{2}{*}{$\begin{array}{l}\text { m.p }{ }^{\circ} \mathbf{C} \\
\text { Yield\% }\end{array}$} & \multirow[b]{2}{*}{$\begin{array}{l}\text { M. F } \\
\text { M.wt }\end{array}$} & \multicolumn{3}{|c|}{ Analysis Calcd/Found \% } \\
\hline & & & & & C & $\mathbf{H}$ & N \\
\hline $2 a$ & $\mathrm{C}_{8} \mathrm{H}_{17}$ & & $\begin{array}{l}65-67 \\
(69 \%)\end{array}$ & $\begin{array}{c}\mathrm{C}_{12} \mathrm{H}_{22} \mathrm{O}_{3} \\
214.31\end{array}$ & $\begin{array}{l}67.26 \\
67.32\end{array}$ & $\begin{array}{l}10.35 \\
10.41\end{array}$ & \\
\hline $2 b$ & $\mathrm{C}_{12} \mathrm{H}_{25}$ & & $\begin{array}{l}63-65 \\
(75 \%)\end{array}$ & $\begin{array}{c}\mathrm{C}_{16} \mathrm{H}_{30} \mathrm{O}_{3} \\
270.24\end{array}$ & $\begin{array}{l}71.07 \\
71.12\end{array}$ & $\begin{array}{l}11.18 \\
11.24\end{array}$ & \\
\hline $2 c$ & $\mathrm{C}_{18} \mathrm{H}_{37}$ & & $\begin{array}{l}66-68 \\
(70 \%)\end{array}$ & $\begin{array}{c}\mathrm{C}_{22} \mathrm{H}_{42} \mathrm{O}_{3} \\
354.58\end{array}$ & $\begin{array}{l}74.52 \\
74.57\end{array}$ & $\begin{array}{l}11.94 \\
11.99\end{array}$ & \\
\hline $3 a$ & $\mathrm{C}_{8} \mathrm{H}_{17}$ & $-H$ & $\begin{array}{c}120-122 \\
(76 \%)\end{array}$ & $\begin{array}{c}\mathrm{C}_{14} \mathrm{H}_{27} \mathrm{NO}_{5} \\
289.37\end{array}$ & $\begin{array}{l}58.11 \\
58.16\end{array}$ & $\begin{array}{l}9.40 \\
9.45\end{array}$ & $\begin{array}{l}4.84 \\
4.89\end{array}$ \\
\hline $3 b$ & $\mathrm{C}_{12} \mathrm{H}_{25}$ & $-\mathrm{H}$ & $\begin{array}{c}125-127 \\
(74 \%)\end{array}$ & $\begin{array}{c}\mathrm{C}_{18} \mathrm{H}_{35} \mathrm{NO}_{5} \\
345.48\end{array}$ & $\begin{array}{c}62.586 \\
2.63\end{array}$ & $\begin{array}{c}10.211 \\
0.25\end{array}$ & $\begin{array}{l}4.05 \\
4.09\end{array}$ \\
\hline $3 c$ & $\mathrm{C}_{18} \mathrm{H}_{37}$ & $-\mathrm{H}$ & $\begin{array}{c}123-125 \\
(78 \%)\end{array}$ & $\begin{array}{c}\mathrm{C}_{24} \mathrm{H}_{47} \mathrm{NO}_{5} \\
429.65\end{array}$ & $\begin{array}{l}67.09 \\
67.13\end{array}$ & $\begin{array}{c}11.031 \\
1.08\end{array}$ & $\begin{array}{l}3.26 \\
3.30\end{array}$ \\
\hline $4 a$ & $\mathrm{C}_{8} \mathrm{H}_{17}$ & $-\mathrm{CH}_{3}$ & $\begin{array}{l}90-92 \\
(77 \%)\end{array}$ & $\begin{array}{c}\mathrm{C}_{15} \mathrm{H}_{29} \mathrm{NO}_{5} \\
303.40\end{array}$ & $\begin{array}{l}59.38 \\
59.43\end{array}$ & $\begin{array}{l}9.63 \\
9.68\end{array}$ & $\begin{array}{l}4.62 \\
4.67\end{array}$ \\
\hline $4 b$ & $\mathrm{C}_{12} \mathrm{H}_{25}$ & $-\mathrm{CH}_{3}$ & $\begin{array}{l}90-92 \\
(68 \%)\end{array}$ & $\begin{array}{c}\mathrm{C}_{19} \mathrm{H}_{37} \mathrm{NO}_{5} \\
359.51\end{array}$ & $\begin{array}{l}63.48 \\
63.52\end{array}$ & $\begin{array}{c}10.371 \\
0.42\end{array}$ & $\begin{array}{l}3.90 \\
3.95\end{array}$ \\
\hline $4 \mathrm{c}$ & $\mathrm{C}_{18} \mathrm{H}_{37}$ & $-\mathrm{CH}_{3}$ & $\begin{array}{l}91-93 \\
(65 \%)\end{array}$ & $\begin{array}{c}\mathrm{C}_{25} \mathrm{H}_{49} \mathrm{NO}_{5} \\
443.67\end{array}$ & $\begin{array}{c}67.686 \\
7.73\end{array}$ & $\begin{array}{c}11.131 \\
1.17\end{array}$ & $\begin{array}{l}3.16 \\
3.20\end{array}$ \\
\hline $5 a$ & $\mathrm{C}_{8} \mathrm{H}_{17}$ & $\mathrm{CH}\left(\mathrm{CH}_{3}\right)_{2}$ & $\begin{array}{l}85-87 \\
(75 \%)\end{array}$ & $\begin{array}{c}\mathrm{C}_{17} \mathrm{H}_{33} \mathrm{NO}_{5} \\
331.46\end{array}$ & $\begin{array}{l}61.60 \\
61.65\end{array}$ & $\begin{array}{l}10.04 \\
10.09\end{array}$ & $\begin{array}{l}4.23 \\
4.27\end{array}$ \\
\hline $5 b$ & $\mathrm{C}_{12} \mathrm{H}_{25}$ & $\mathrm{CH}\left(\mathrm{CH}_{3}\right)_{2}$ & $\begin{array}{l}80-82 \\
(70 \%)\end{array}$ & $\begin{array}{c}\mathrm{C}_{21} \mathrm{H}_{41} \mathrm{NO}_{5} \\
387.56\end{array}$ & $\begin{array}{c}65.086 \\
5.12\end{array}$ & $\begin{array}{c}10.661 \\
0.70\end{array}$ & $\begin{array}{l}3.61 \\
3.65\end{array}$ \\
\hline $5 c$ & $\mathrm{C}_{18} \mathrm{H}_{37}$ & $\mathrm{CH}\left(\mathrm{CH}_{3}\right)_{2}$ & $\begin{array}{l}88-90 \\
(71 \%)\end{array}$ & $\begin{array}{c}\mathrm{C}_{27} \mathrm{H}_{53} \mathrm{NO}_{5} \\
471.73\end{array}$ & $\begin{array}{c}68.756 \\
8.79\end{array}$ & $\begin{array}{c}11.321 \\
1.37\end{array}$ & $\begin{array}{l}2.97 \\
3.02\end{array}$ \\
\hline $6 a$ & $\mathrm{C}_{8} \mathrm{H}_{17}$ & $\begin{array}{r}\mathrm{CH}_{3} \mathrm{CH}_{2} \mathrm{CH}- \\
\mathrm{CH} \\
\mathrm{CH}_{3}\end{array}$ & $\begin{array}{l}86-88 \\
(65 \%)\end{array}$ & $\begin{array}{c}\mathrm{C}_{18} \mathrm{H}_{35} \mathrm{NO}_{5} \\
345.48\end{array}$ & $\begin{array}{l}62.58 \\
62.63\end{array}$ & $\begin{array}{c}10.211 \\
0.26\end{array}$ & $\begin{array}{l}4.05 \\
4.09\end{array}$ \\
\hline $6 b$ & $\mathrm{C}_{12} \mathrm{H}_{25}$ & $\mathrm{CH}_{3} \mathrm{CH}_{2} \mathrm{CH}-$ & $\begin{array}{l}85-87 \\
(68 \%)\end{array}$ & $\begin{array}{c}\mathrm{C}_{22} \mathrm{H}_{43} \mathrm{NO}_{5} \\
401.59\end{array}$ & $\begin{array}{c}65.806 \\
5.85\end{array}$ & $\begin{array}{c}10.791 \\
0.83\end{array}$ & $\begin{array}{l}3.49 \\
3.53\end{array}$ \\
\hline $6 c$ & $\mathrm{C}_{18} \mathrm{H}_{37}$ & $\begin{array}{r}\mathrm{CH}_{3} \mathrm{CH}_{2} \mathrm{CH}- \\
\mathrm{I} \\
\mathrm{CH}_{3}\end{array}$ & $\begin{array}{l}79-81 \\
(66 \%)\end{array}$ & $\begin{array}{c}\mathrm{C}_{28} \mathrm{H}_{55} \mathrm{NO}_{5} \\
485.75\end{array}$ & $\begin{array}{c}69.246 \\
9.29 \\
\end{array}$ & $\begin{array}{c}11.411 \\
1.45 \\
\end{array}$ & $\begin{array}{l}2.88 \\
2.94 \\
\end{array}$ \\
\hline $7 a$ & $\mathrm{C}_{8} \mathrm{H}_{17}$ & $\mathrm{C}_{6} \mathrm{H}_{5} \mathrm{CH}_{2}-$ & $\begin{array}{c}105-107 \\
(74 \%)\end{array}$ & $\begin{array}{c}\mathrm{C}_{21} \mathrm{H}_{33} \mathrm{NO}_{5} \\
379.24\end{array}$ & $\begin{array}{l}66.46 \\
66.50\end{array}$ & $\begin{array}{l}8.76 \\
8.81\end{array}$ & $\begin{array}{l}3.96 \\
4.00\end{array}$ \\
\hline $7 b$ & $\mathrm{C}_{12} \mathrm{H}_{25}$ & $\mathrm{C}_{6} \mathrm{H}_{5} \mathrm{CH}_{2}-$ & $\begin{array}{c}106-108 \\
(77 \%)\end{array}$ & $\begin{array}{c}\mathrm{C}_{25} \mathrm{H}_{41} \mathrm{NO}_{5} \\
435.3\end{array}$ & $\begin{array}{l}68.93 \\
68.98\end{array}$ & $\begin{array}{l}9.49 \\
9.54\end{array}$ & $\begin{array}{l}3.22 \\
3.27\end{array}$ \\
\hline 7c & $\mathrm{C}_{18} \mathrm{H}_{37}$ & $\mathrm{C}_{6} \mathrm{H}_{5} \mathrm{CH}_{2}-$ & $\begin{array}{c}109-111 \\
(69 \%)\end{array}$ & $\begin{array}{c}\mathrm{C}_{31} \mathrm{H}_{53} \mathrm{NO}_{5} \\
519.39\end{array}$ & $\begin{array}{l}71.64 \\
71.69\end{array}$ & $\begin{array}{l}10.28 \\
10.33\end{array}$ & $\begin{array}{l}2.96 \\
3.01\end{array}$ \\
\hline $8 a$ & $\mathrm{C}_{8} \mathrm{H}_{17}$ & $p-\mathrm{OHC}_{6} \mathrm{H}_{5} \mathrm{CH}_{2}$ & $\begin{array}{c}105-107 \\
(65 \%)\end{array}$ & $\begin{array}{c}\mathrm{C}_{21} \mathrm{H}_{33} \mathrm{NO}_{6} \\
395.23\end{array}$ & $\begin{array}{l}63.78 \\
63.83\end{array}$ & $\begin{array}{l}8.41 \\
8.48\end{array}$ & $\begin{array}{l}3.54 \\
3.60\end{array}$ \\
\hline $8 b$ & $\mathrm{C}_{12} \mathrm{H}_{25}$ & $p-\mathrm{OHC}_{6} \mathrm{H}_{5} \mathrm{CH}_{2}$ & $\begin{array}{c}108-110 \\
(60 \%)\end{array}$ & $\begin{array}{c}\mathrm{C}_{25} \mathrm{H}_{41} \mathrm{NO}_{6} \\
451.29\end{array}$ & $\begin{array}{l}66.49 \\
66.54\end{array}$ & $\begin{array}{l}9.15 \\
9.20\end{array}$ & $\begin{array}{l}3.10 \\
3.16\end{array}$ \\
\hline $8 c$ & $\mathrm{C}_{18} \mathrm{H}_{37}$ & $p-\mathrm{OHC}_{6} \mathrm{H}_{5} \mathrm{CH}_{2}$ & $\begin{array}{c}115-117 \\
(74 \%)\end{array}$ & $\begin{array}{c}\mathrm{C}_{31} \mathrm{H}_{53} \mathrm{NO}_{6} \\
535.39\end{array}$ & $\begin{array}{l}69.50 \\
69.55\end{array}$ & $\begin{array}{c}9.97 \\
10.02\end{array}$ & $\begin{array}{l}2.61 \\
2.66\end{array}$ \\
\hline $9 a$ & $\mathrm{C}_{8} \mathrm{H}_{17}$ & $\mathrm{HOCH}_{2}$ & $\begin{array}{c}140-142 \\
(61 \%)\end{array}$ & $\begin{array}{c}\mathrm{C}_{15} \mathrm{H}_{29} \mathrm{NO}_{6} \\
319.39\end{array}$ & $\begin{array}{l}56.41 \\
56.44\end{array}$ & $\begin{array}{l}9.15 \\
9.19\end{array}$ & $\begin{array}{l}4.39 \\
4.43\end{array}$ \\
\hline $9 b$ & $\mathrm{C}_{12} \mathrm{H}_{25}$ & $\mathrm{HOCH}_{2}$ & $\begin{array}{c}139-141 \\
(60 \%)\end{array}$ & $\begin{array}{c}\mathrm{C}_{19} \mathrm{H}_{37} \mathrm{NO}_{6} \\
375.26\end{array}$ & $\begin{array}{l}60.77 \\
60.82\end{array}$ & $\begin{array}{l}9.93 \\
9.98\end{array}$ & $\begin{array}{l}3.73 \\
3.77\end{array}$ \\
\hline $9 c$ & $\mathrm{C}_{18} \mathrm{H}_{37}$ & $\mathrm{HOCH}_{2}$ & $\begin{array}{c}128-140 \\
(66 \%)\end{array}$ & $\begin{array}{c}\mathrm{C}_{25} \mathrm{H}_{49} \mathrm{NO}_{6} \\
459.36\end{array}$ & $\begin{array}{l}65.32 \\
65.37\end{array}$ & $\begin{array}{l}10.74 \\
10.78\end{array}$ & $\begin{array}{l}3.05 \\
3.09\end{array}$ \\
\hline
\end{tabular}


Table 1. (cont.)

Physical data of the synthesized compounds

\begin{tabular}{|c|c|c|c|c|c|c|c|}
\hline \multirow[b]{2}{*}{ Compds } & \multirow[b]{2}{*}{$\mathbf{R}$} & \multirow[b]{2}{*}{$\mathbf{R}^{\prime}$} & \multirow[b]{2}{*}{$\begin{array}{l}\text { m.p }{ }^{\circ} \mathbf{C} \\
\text { Yield\% }\end{array}$} & \multirow[b]{2}{*}{$\begin{array}{l}\text { M. F } \\
\text { M.wt }\end{array}$} & \multicolumn{3}{|c|}{ Analysis Calcd/Found \% } \\
\hline & & & & & C & $\mathbf{H}$ & $\mathbf{N}$ \\
\hline $10 a$ & $\mathrm{C}_{8} \mathrm{H}_{17}$ & $\begin{array}{r}\mathrm{CH}_{3} \mathrm{CH}- \\
\mathrm{O} \\
\mathrm{OH}\end{array}$ & $\begin{array}{c}120-122 \\
(70 \%)\end{array}$ & $\begin{array}{c}\mathrm{C}_{16} \mathrm{H}_{31} \mathrm{NO}_{6} \\
333.22\end{array}$ & $\begin{array}{l}57.64 \\
57.68\end{array}$ & $\begin{array}{l}9.37 \\
9.41\end{array}$ & $\begin{array}{l}4.20 \\
4.24\end{array}$ \\
\hline $10 b$ & $\mathrm{C}_{12} \mathrm{H}_{25}$ & $\begin{array}{r}\mathrm{CH}_{3} \mathrm{CH}- \\
\mathrm{OH}\end{array}$ & $\begin{array}{c}118-120 \\
(75 \%)\end{array}$ & $\begin{array}{c}\mathrm{C}_{20} \mathrm{H}_{39} \mathrm{NO}_{6} \\
389.28\end{array}$ & $\begin{array}{l}61.67 \\
61.71\end{array}$ & $\begin{array}{l}10.09 \\
10.14\end{array}$ & $\begin{array}{l}3.60 \\
3.66\end{array}$ \\
\hline $10 c$ & $\mathrm{C}_{18} \mathrm{H}_{37}$ & $\begin{array}{c}\mathrm{CH}_{3} \mathrm{CH}- \\
\mathrm{OH}\end{array}$ & $\begin{array}{c}121-123 \\
(74 \%)\end{array}$ & $\begin{array}{c}\mathrm{C}_{26} \mathrm{H}_{51} \mathrm{NO}_{6} \\
473.37\end{array}$ & $\begin{array}{l}65.93 \\
65.98 \\
\end{array}$ & $\begin{array}{l}10.85 \\
10.88 \\
\end{array}$ & $\begin{array}{l}2.96 \\
2.99 \\
\end{array}$ \\
\hline $11 a$ & $\mathrm{C}_{8} \mathrm{H}_{17}$ & $\mathrm{HOOC}-\mathrm{CH}_{2}$ & $\begin{array}{c}150-152 \\
(60 \%)\end{array}$ & $\begin{array}{c}\mathrm{C}_{16} \mathrm{H}_{29} \mathrm{NO}_{7} \\
347.19\end{array}$ & $\begin{array}{l}55.32 \\
55.37\end{array}$ & $\begin{array}{l}8.41 \\
8.44\end{array}$ & $\begin{array}{l}4.03 \\
4.08\end{array}$ \\
\hline $11 b$ & $\mathrm{C}_{12} \mathrm{H}_{25}$ & $\mathrm{HOOC}-\mathrm{CH}_{2}$ & $\begin{array}{c}152-154 \\
(65 \%)\end{array}$ & $\begin{array}{c}\mathrm{C}_{20} \mathrm{H}_{37} \mathrm{NO}_{7} \\
403.26\end{array}$ & $\begin{array}{l}59.53 \\
59.57\end{array}$ & $\begin{array}{l}9.24 \\
9.29\end{array}$ & $\begin{array}{l}3.47 \\
3.52\end{array}$ \\
\hline $11 \mathrm{c}$ & $\mathrm{C}_{18} \mathrm{H}_{37}$ & $\mathrm{HOOC}-\mathrm{CH}_{2}$ & $\begin{array}{c}150-152 \\
(75)\end{array}$ & $\begin{array}{c}\mathrm{C}_{26} \mathrm{H}_{49} \mathrm{NO}_{7} \\
487.35\end{array}$ & $\begin{array}{l}64.03 \\
64.08\end{array}$ & $\begin{array}{l}10.13 \\
10.17\end{array}$ & $\begin{array}{l}2.87 \\
2.90\end{array}$ \\
\hline $12 \mathrm{a}$ & $\mathrm{C}_{8} \mathrm{H}_{17}$ & $\mathrm{O}-\mathrm{NH}_{2} \mathrm{C}_{6} \mathrm{H}_{5} \mathrm{COOH}$ & $\begin{array}{c}130-132 \\
(68 \%)\end{array}$ & $\begin{array}{c}\mathrm{C}_{19} \mathrm{H}_{29} \mathrm{NO}_{5} \\
351.45\end{array}$ & $\begin{array}{l}64.93 \\
64.98\end{array}$ & $\begin{array}{l}8.32 \\
8.37\end{array}$ & $\begin{array}{l}3.99 \\
4.05\end{array}$ \\
\hline $12 b$ & $\mathrm{C}_{12} \mathrm{H}_{25}$ & $\mathrm{O}-\mathrm{NH}_{2} \mathrm{C}_{6} \mathrm{H}_{5} \mathrm{COOH}$ & $\begin{array}{c}133-135 \\
(73 \%)\end{array}$ & $\begin{array}{c}\mathrm{C}_{23} \mathrm{H}_{37} \mathrm{NO}_{5} \\
407.56\end{array}$ & $\begin{array}{l}67.78 \\
67.83\end{array}$ & $\begin{array}{l}9.15 \\
9.19\end{array}$ & $\begin{array}{l}3.44 \\
3.49\end{array}$ \\
\hline $12 c$ & $\mathrm{C}_{18} \mathrm{H}_{37}$ & $\mathrm{o}-\mathrm{NH}_{2} \mathrm{C}_{6} \mathrm{H}_{5} \mathrm{COOH}$ & $\begin{array}{c}130-132 \\
(75)\end{array}$ & $\begin{array}{c}\mathrm{C}_{29} \mathrm{H}_{49} \mathrm{NO}_{5} \\
491.72\end{array}$ & $\begin{array}{l}70.84 \\
70.88\end{array}$ & $\begin{array}{l}10.04 \\
10.09\end{array}$ & $\begin{array}{l}2.85 \\
2.89\end{array}$ \\
\hline
\end{tabular}

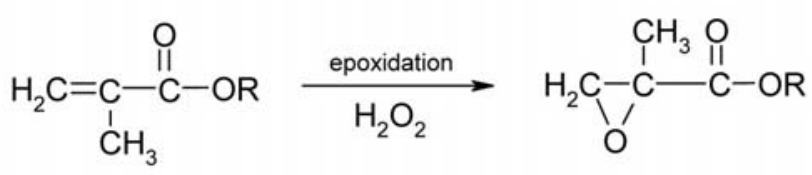

$(1 \mathrm{a}-\mathrm{c})$

$(2 a-c)$

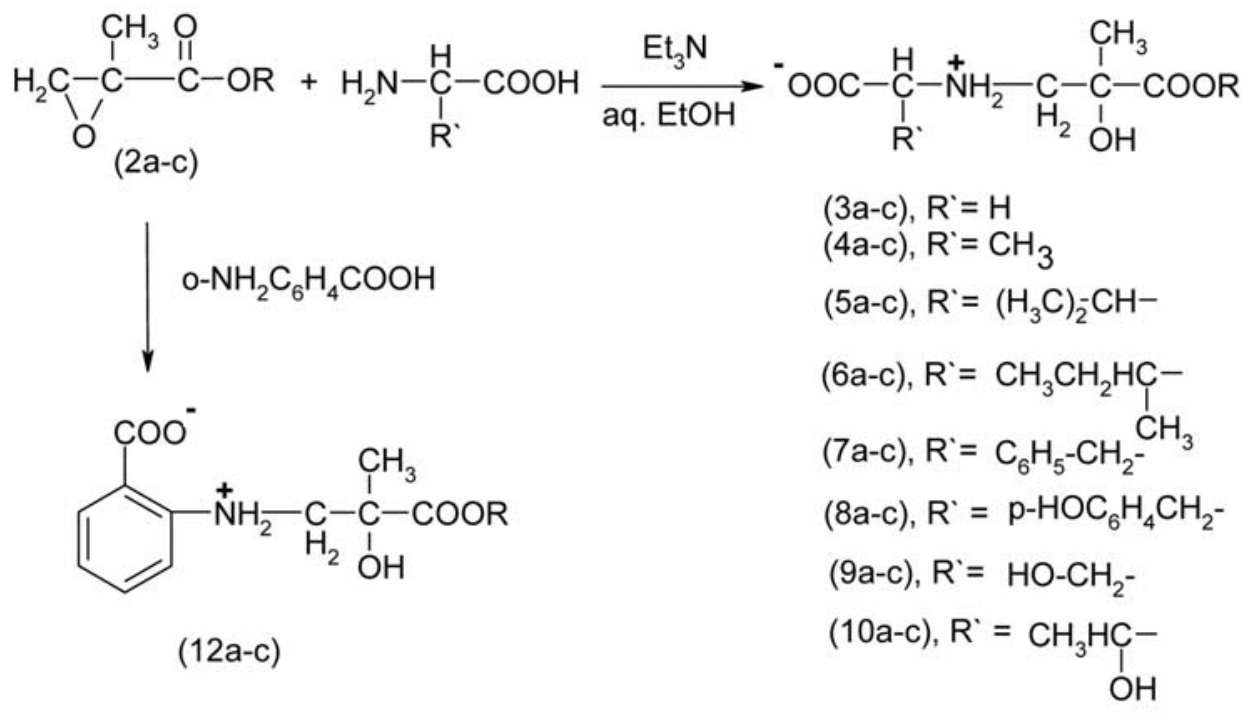

a; $\mathrm{R}=\mathrm{C}_{8} \mathrm{H}_{17} \quad \mathrm{~b} ; \mathrm{R}=\mathrm{C}_{12} \mathrm{H}_{25} \quad \mathrm{C} ; \mathrm{R}=\mathrm{C}_{18} \mathrm{H}_{37} \quad(11 \mathrm{a}-\mathrm{c}), \mathrm{R}^{\prime}=\mathrm{HOOC}^{-\mathrm{CH}_{2}-}$ 
Table 2.

Spectral data of some synthesized compounds

\begin{tabular}{|c|c|c|c|}
\hline Compds & IR: $v\left(\mathrm{~cm}^{-1}\right)$ & ${ }^{1} \mathrm{H} \mathrm{NMR}\left(\mathrm{CDCl}_{3}\right): \delta$ & MS: $\mathrm{m} / \mathbf{z}(\%)$ \\
\hline $2 b$ & $\begin{array}{c}2920-2850\left(\mathrm{CH}_{2} \text { aliphatic }\right) \\
1720(\mathrm{C}=\mathrm{O}) \text { and } 1270(\mathrm{C}-\mathrm{O})\end{array}$ & $\begin{array}{c}0.9\left(\mathrm{t}, 3 \mathrm{H}, \mathrm{CH}_{3} \text { terminal of alkyl chain), } 1.2\left(\mathrm{~m}, 2 \mathrm{OH}, \mathrm{CH}_{2} \text { of }\right.\right. \\
\text { alkyl chain), } 1.4\left(\mathrm{~s}, 3 \mathrm{H}, \mathrm{CH}_{3} \text { of } \alpha \text {-substituted ester), } 3.1\right. \\
\left(\mathrm{s}, 2 \mathrm{H}, \mathrm{CH}_{2} \text { of oxirane ring) and } 4.2\left(\mathrm{t}, 2 \mathrm{H}, \mathrm{COOCH}{ }_{2}\right)\right.\end{array}$ & \\
\hline $3 c$ & $\begin{array}{c}3420(\mathrm{OH}), 3320(\mathrm{NH}), 2910-2830 \\
\left(\mathrm{CH}_{2} \text { aliphatic }\right), 1730(\mathrm{C}=\mathrm{O}) \\
\text { and } 1300(\mathrm{C}-\mathrm{O})\end{array}$ & $\begin{array}{c}0.96\left(\mathrm{t}, 3 \mathrm{H}, \mathrm{CH}_{3} \text { terminal } \mathrm{CH}_{3} \text { of alkyl chain), } 1.3(\mathrm{~m}, 32 \mathrm{H},\right. \\
\mathrm{CH}_{2} \text { of alkyl chain), } 1.6\left(\mathrm{~s}, 3 \mathrm{H}, \mathrm{CH}_{3} \text { of } \alpha \text {-substituted }\right. \\
\text { esters), 2.0-2.2 (broad, } 2 \mathrm{H}, \mathrm{NH} \text { and alcoholic } \mathrm{OH}), 2.9 \\
(\mathrm{~s}, 2 \mathrm{H}, \underset{\mathrm{NHCH}}{2}), 3.5\left(\mathrm{~s}, 2 \mathrm{H}, \mathrm{CH}_{2} \mathrm{COOH}\right), 4.1(\mathrm{t}, 2 \mathrm{H}, \\
\left.\mathrm{COOC \textrm {CH } _ { 2 }}\right) \text { and } 11.2(\mathrm{~s}, 1 \mathrm{H}, \mathrm{COOH})\end{array}$ & $\mathrm{M}^{+}+1=430(30)$ \\
\hline $4 a$ & $\begin{array}{c}3450(\mathrm{OH}), 3340(\mathrm{NH}), 2950-2820 \\
\left(\mathrm{CH}_{2} \text { aliphatic }\right), 1710(\mathrm{C}=\mathrm{O}) \text { and } \\
1280(\mathrm{C}-\mathrm{O})\end{array}$ & 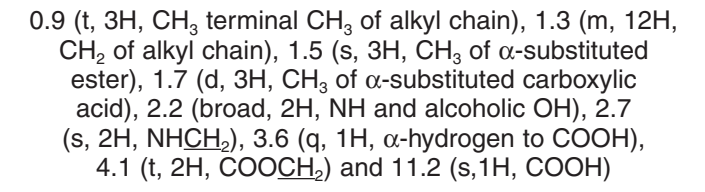 & $\mathrm{M}^{+}=303(25)$ \\
\hline $5 b$ & $\begin{array}{c}3420(\mathrm{OH}), 3300(\mathrm{NH}), 2950-2820 \\
\left(\mathrm{CH}_{2} \text { aliphatic }\right), 1700(\mathrm{C}=\mathrm{O}) \\
\text { and } 1270(\mathrm{C}-\mathrm{O})\end{array}$ & $\begin{array}{c}0.9\left(\mathrm{t}, 3 \mathrm{H}, \mathrm{CH}_{3} \text { terminal } \mathrm{CH}_{3} \text { of alkyl chain), } 1.1(\mathrm{~d}, 6 \mathrm{H},\right. \\
2 \mathrm{CH}_{3} \text { of isopropyl moiety), } 1.3\left(\mathrm{~m}, 2 \mathrm{OH}, \mathrm{CH}_{2} \text { of alkyl }\right. \\
\text { chain), } 1.4\left(\mathrm{~s}, 3 \mathrm{H}, \mathrm{CH}_{3} \text { of } \alpha \text {-substituted ester }\right), 2.2(\text { broad, } \\
2 \mathrm{H}, \mathrm{NH} \text { and alcoholic } \mathrm{OH}), 2.5\left(\mathrm{~m}, 1 \mathrm{H}, \mathrm{CH}\left(\mathrm{CH}_{3}\right)_{2}\right) \\
2.8\left(\mathrm{~s}, 2 \mathrm{H}, \mathrm{NH} \underline{\mathrm{CH}}_{2}\right), 3.5(\mathrm{~d}, 1 \mathrm{H}, \alpha \text {-hydrogen to } \mathrm{COOH}) \\
4.0\left(\mathrm{t}, 2 \mathrm{H}, \mathrm{COOCH}_{2}\right) \text { and } 11.0(\mathrm{~s}, 1 \mathrm{H}, \mathrm{COOH})\end{array}$ & \\
\hline $6 c$ & $\begin{array}{c}3450(\mathrm{OH}), 3340(\mathrm{NH}), 2950-2820 \\
\left(\mathrm{CH}_{2} \text { aliphatic }\right), 1710(\mathrm{C}=\mathrm{O}) \\
\text { and } 1275(\mathrm{C}-\mathrm{O})\end{array}$ & $\begin{array}{c}\text { 0.9-1.1 (m, } 9 \mathrm{H}, 3 \mathrm{CH}_{3} \text { (terminal } \mathrm{CH}_{3} \text { of alkyl chain and } \\
2 \mathrm{CH}_{3} \text { of amino acid residue), } 1.2\left(\mathrm{~m}, 32 \mathrm{H}, \mathrm{CH}_{2} \text { of alkyl }\right. \\
\text { chain), } 1.3\left(\mathrm{~m}, 2 \mathrm{H}, \mathrm{CH}_{2} \text { of alkyl chain of amino acid }\right. \\
\text { residue), } 1.5\left(\mathrm{~s}, 3 \mathrm{H}, \mathrm{CH}_{3} \text { of } \alpha \text {-substituted ester), } 2.2\right. \\
\text { (broad, } 2 \mathrm{H}, \mathrm{NH} \text { and alcoholic } \mathrm{OH}), 2.5(\mathrm{~m}, 1 \mathrm{H}, \underline{\mathrm{CH}} \\
\text { of alkyl chain of amino acid), } 3.0\left(\mathrm{~s}, 2 \mathrm{H}, \mathrm{NH} \underline{\mathrm{CH}}_{2}\right), \\
\quad 3.5(\mathrm{~d}, 1 \mathrm{H}, \alpha-h y d r o g e n \text { to } \mathrm{COOH}), 4.1(\mathrm{t}, 2 \mathrm{H}, \\
\left.\mathrm{COOCH} \mathrm{CH}_{2}\right) \text { and } 11.0(\mathrm{~s}, 1 \mathrm{H}, \mathrm{COOH})\end{array}$ & $M^{+}-2=483(35)$ \\
\hline $7 a$ & $\begin{array}{l}3430(\mathrm{OH}), 3220(\mathrm{NH}), 3006 \\
(\mathrm{CH} \text { aromatic }), 2930-2810 \\
\left(\mathrm{CH}_{2} \text { aliphatic), } 1730(\mathrm{C}=\mathrm{O})\right. \\
\text { and } 1285(\mathrm{C}-\mathrm{O})\end{array}$ & $\begin{array}{c}0.9 \text { (t, } 3 \mathrm{H}, \mathrm{CH}_{3} \text { terminal } \mathrm{CH}_{3} \text { of alkyl chain), } 1.3(\mathrm{~m}, 12 \mathrm{H}, \\
\mathrm{CH}_{2} \text { of alkyl chain), } 1.5\left(\mathrm{~s}, 3 \mathrm{H}, \mathrm{CH}_{3} \text { of } \alpha \text {-substituted esters), }\right. \\
2.1(\text { broad, } 2 \mathrm{H}, \mathrm{NH} \text { and alcoholic } \mathrm{OH}), 2.7 \text { (s, } 2 \mathrm{H}, \\
\left.\mathrm{NH}_{2} \underline{\mathrm{CH}}_{2}\right), 3.1\left(\mathrm{~d}, 2 \mathrm{H}, \mathrm{CH}_{2}-\mathrm{Ph}\right), 3.7(\mathrm{t}, 1 \mathrm{H}, \alpha \text {-hydrogen } \\
\text { to } \mathrm{COOH}), 4.1\left(\mathrm{t}, 2 \mathrm{H}, \mathrm{COOCH} \mathrm{CH}_{2}\right), 7.1-7.3(\mathrm{~m}, 5 \mathrm{H}, \\
\text { ArH) and } 11.0(\mathrm{~s}, 1 \mathrm{H}, \mathrm{COOH})\end{array}$ & $\mathrm{M}^{+}=379(22)$ \\
\hline $8 c$ & $\begin{array}{l}3450(\mathrm{OH}), 3200(\mathrm{NH}), 3050 \\
(\mathrm{CH} \text { aromatic }), 2950-2820 \\
\left(\mathrm{CH}_{2} \text { aliphatic }\right), 1720(\mathrm{C}=\mathrm{O}) \\
\text { and } 1300(\mathrm{C}-\mathrm{O})\end{array}$ & 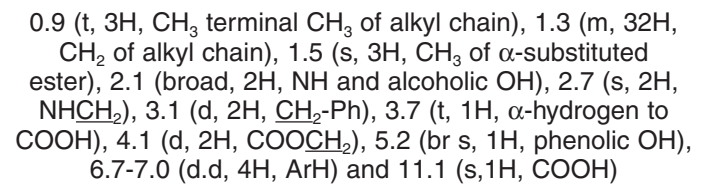 & $\mathrm{M}^{+}+2=537(42)$ \\
\hline $9 b$ & $\begin{array}{l}3430-3250(\mathrm{OH}) \text { and }(\mathrm{NH}) \\
2950-2820\left(\mathrm{CH}_{2} \text { aliphatic }\right), 1710 \\
(\mathrm{C}=\mathrm{O}) \text { and } 1270(\mathrm{C}-\mathrm{O})\end{array}$ & $\begin{array}{c}0.9\left(\mathrm{t}, 3 \mathrm{H}, \mathrm{CH}_{3} \text { terminal } \mathrm{CH}_{3} \text { of alkyl chain), } 1.3(\mathrm{~m}, 12 \mathrm{H},\right. \\
\mathrm{CH}_{2} \text { of alkyl chain), } 1.5\left(\mathrm{~s}, 3 \mathrm{H}, \mathrm{CH}_{3} \text { of } \alpha \text {-substituted }\right. \\
\text { esters), } 2.2 \text { (broad, } 3 \mathrm{H}, \mathrm{NH} \text { and } 2(\text { alcoholic } \mathrm{OH}), 3.0 \\
\left(\mathrm{~s}, 2 \mathrm{H}, \mathrm{NH} \mathrm{CH}_{2}\right), 3.6(\mathrm{t}, 1 \mathrm{H}, \mathrm{CHCOOH}), 3.9(\mathrm{~d}, 2 \mathrm{H}, \\
\left.\mathrm{CH}_{2}-\mathrm{OH}\right), 4.2\left(\mathrm{t}, 2 \mathrm{H}, \mathrm{COOCH}{ }_{2}\right) \text {, and } 11.2(\mathrm{~s}, 1 \mathrm{H}, \mathrm{COOH})\end{array}$ & \\
\hline $10 a$ & $\begin{array}{l}3420-3230(\mathrm{OH}) \text { and }(\mathrm{NH}) \\
2950-2820\left(\mathrm{CH}_{2} \text { aliphatic }\right), 1710 \\
(\mathrm{C}=\mathrm{O}) \text { and } 1270(\mathrm{C}-\mathrm{O})\end{array}$ & $\begin{array}{c}0.9\left(\mathrm{t}, 3 \mathrm{H}, \mathrm{CH}_{3} \text { terminal } \mathrm{CH}_{3} \text { of alkyl chain), } 1.1(\mathrm{~d}, 3 \mathrm{H} \text {, }\right. \\
\left.\mathrm{CH}_{3} \text { in amino acid residue }\right), 1.4\left(\mathrm{~m}, 12 \mathrm{H}, \mathrm{CH}_{2} \text { of alkyl }\right. \\
\text { chain), } 1.6\left(\mathrm{~s}, 3 \mathrm{H}, \mathrm{CH}_{3} \text { of } \alpha \text {-substituted ester), } 2.1 \text { (broad, }\right. \\
3 \mathrm{H}, \mathrm{NH} \text { and alcoholic } 2 \mathrm{OH}), 3.0\left(\mathrm{~s}, 2 \mathrm{H}, \mathrm{NH}^{-} \mathrm{CH}_{2}\right), 3.4 \\
(\mathrm{~d}, 1 \mathrm{H}, \alpha \text {-hydrogen to } \mathrm{COOH}), 3.9\left(\mathrm{~m}, 1 \mathrm{H}, \mathrm{CH}_{3}-\mathrm{CH}-\mathrm{OH}\right. \\
\text { of amino acid part), } 4.2\left(\mathrm{t}, 2 \mathrm{H}, \mathrm{COO} \mathrm{CH}_{2}\right) \text { and } 11.1 \\
(\mathrm{~s}, 1 \mathrm{H}, \mathrm{COOH})\end{array}$ & $\mathrm{M}^{+}=333(32)$ \\
\hline $11 c$ & $\begin{array}{c}3420-3220(\mathrm{OH}) \text { and }(\mathrm{NH}) \\
2940-2830\left(\mathrm{CH}_{2} \text { aliphatic }\right), 1710 \\
(\mathrm{C}=\mathrm{O}) \text { and } 1280(\mathrm{C}-\mathrm{O})\end{array}$ & $\begin{array}{c}0.9 \text { (t, } 3 \mathrm{H}, \mathrm{CH}_{3} \text { terminal } \mathrm{CH}_{3} \text { of alkyl chain), } 1.3(\mathrm{~m}, 32 \mathrm{H} \\
\mathrm{CH}_{2} \text { of alkyl chain), } 1.6\left(\mathrm{~s}, 3 \mathrm{H}, \mathrm{CH}_{3} \text { of } \alpha \text {-substituted ester) }\right. \\
2.1(\text { broad, } 2 \mathrm{H}, \mathrm{NH} \text { and alcoholic } \mathrm{OH}), 2.6(\mathrm{~d}, 2 \mathrm{H} \\
\left.\frac{\left.\mathrm{CH}_{2}-\mathrm{COOH}\right), 2.9\left(\mathrm{~s}, 2 \mathrm{H}, \mathrm{NH} \mathrm{CH}_{2}\right), 3.8(\mathrm{t}, 1 \mathrm{H}, \alpha \text {-hydrogen }}{\text { to other } \mathrm{COOH}), 4.1(\mathrm{t}, 2 \mathrm{H}, \mathrm{COOCH}}\right) \text { and } 11.2 \text { (br s, } \\
2 \mathrm{H}, 2 \mathrm{COOH})\end{array}$ & \\
\hline $12 b$ & $\begin{array}{c}3430(\mathrm{OH}), 3220(\mathrm{NH}), 3006 \\
(\mathrm{CH} \text { aromatic }), 2930-2810 \\
\left(\mathrm{CH}_{2} \text { aliphatic }\right), 1730(\mathrm{C}=\mathrm{O}) \\
\text { and } 1285(\mathrm{C}-\mathrm{O})\end{array}$ & $\begin{array}{c}0.9\left(\mathrm{t}, 3 \mathrm{H}, \mathrm{CH}_{3} \text { terminal } \mathrm{CH}_{3} \text { of alkyl chain), } 1.3(\mathrm{~m}, 2 \mathrm{H} \text {, }\right. \\
\mathrm{CH}_{2} \text { of alkyl chain), } 1.6\left(\mathrm{~s}, 3 \mathrm{H}, \mathrm{CH}_{3} \text { of } \alpha \text {-substituted ester), }\right. \\
2.1(\mathrm{~s}, 1 \mathrm{H}, \text { alcoholic } \mathrm{OH}), 3.3\left(\mathrm{~s}, 2 \mathrm{H}, \mathrm{NHCH}_{2}\right), 3.8(\mathrm{br} \mathrm{s}, \\
1 \mathrm{H}, \mathrm{NH}), 4.1\left(\mathrm{t}, 2 \mathrm{H}, \mathrm{CH}_{2}-\mathrm{COOH}\right), 6.5-7.8(\mathrm{~m}, 4 \mathrm{H}, \mathrm{ArH}) \\
\text { and } 11.0(\mathrm{~s}, 1 \mathrm{H}, \mathrm{COOH})\end{array}$ & \\
\hline
\end{tabular}


solution was shaken and heated for $2 \mathrm{hr}$, then allowed to stand overnight at room temperature. Water was then added and the separated solid was crystalized using a suitable solvent to give (2a-c).

\subsection{General procedure of the reaction of epoxy fatty ester with amino acids}

Triethylamine ( 1 mmole) dissolved in an aqueous ethanol solution (65 wt \% ethanol) was added to amino acid (1 mmole) to protect (as a salt) the carboxyl group of the amino acid. The mixture was stirred at room temperature for $20 \mathrm{~min}$. Subsequently, epoxy fatty ester 2 ( 1 mmole) was added using a dropper, and the mixture was stirred at $50{ }^{\circ} \mathrm{C}$ for $8 \mathrm{hr}$ or at $60{ }^{\circ} \mathrm{C}$ for one night. Then the triethylamine and ethanol were evaporated. The residue obtained was washed with water and petroleum ether, then dried under vacuum and crystalized using a suitable solvent to obtain (3a-c to 11a-c).

\subsection{Surface active properties}

\subsubsection{Surface and interfacial tension}

Surface and interfacial tension were measured using Du-Nouy tensiometer (Findly., 1963) (Kruss, Type 8451), with 0.1 wt \% aqueous solution at room temperature $\left(25^{\circ} \mathrm{C}\right)$.

\subsubsection{Kraft point}

The prepared amphoteric surfactants were measured as the temperature where $1 \%$ dispersion becomes clear under gradual heating (Wiel et al., 1963).

\subsubsection{Wetting time}

Wetting time was determined by immersing a sample of cotton fabric in a 1.0 wt \% aqueous solution of surfactants ( Masuyama et al., 1987).

\subsubsection{Foaming power}

Foaming power was measured according to (Somaya et al., 1998). In this procedure a $25 \mathrm{ml}$ solution (1.0 wt \%) was shaken vigorously for 10 seconds in a $100 \mathrm{ml}$ glass stopper, graduated cylinder, at $25^{\circ} \mathrm{C}$. the solution was allowed to stand. The foam height and foaming stability were measured.

\subsubsection{Emulsification stability}

Emulsification stability was tested using $10 \mathrm{ml}$ of a $20 \mathrm{~m} \mathrm{~mol}$. aqueous solution of surfactant and $5 \mathrm{ml}$ of toluene at $40{ }^{\circ} \mathrm{C}$. The emulsifying property was determined as the time its took for an aqueous volume separating from the emulsion layer to reach
$9 \mathrm{ml}$ counting from the moment of the cession shaking (El-Sawy et al., 1991).

\subsubsection{Critical micelle concentration}

(CMC) values for the prepared surfactants were determined by the electrical conductivity method (Takeshi., 1970).

\subsection{7. $\mathrm{Ca}^{++}$stability}

Calcium stability of compounds was determined as described according to (Bristiline et al., 1980).

\subsection{Biodegradability test}

Biodegradability Die-away test in river water of the prepared surfactants (1.0 wt \%) was determined by the surface tension method (Eter et al., 1974) using Du Nouy Tensiometer (Kruss type 8451). Samples taken daily or even more frequently were filtered through Wattmann filter paper number (1) before measuring the surface tension. Surface tension measurements were made periodically each day, on each sample during the degradation test. Biodegradation percent (D) for each sample was calculated using the equation, $D=\left[\left(\gamma_{t}-\gamma_{0}\right) /\left(\gamma_{b t}{ }^{-}\right.\right.$ $\left.\left.\gamma_{0}\right)\right] \times 100$ where $\gamma_{t}=$ surface tension at time $t, \gamma_{0}=$ surface tension at zero time, $\gamma_{\mathrm{bt}}=$ surface tension of blank experiment at time t (without samples).

\subsection{Antimicrobial activity}

The antimicrobial activities of the surfactants were evaluated by the agar dilution method (ElSukkary et al., 1987). Three kinds of Gram-positive bacterial strains, Stophylococcus Aureus; Bacillus Subtiles and Sarcina Lutea, three kinds of Gramnegative bacteria strains, Escherichia Coli, Salmonella Trphi and Pseudomonas Aeruginosa and six kinds of fungal strains, Candida Albicans, Saccharomyces Cerevisiae, Alternaria Humicala, Fusazium Oxysporum, Aspergillus Flavus and Microsporium Gypseum were used for the testes. Nutrient agar and Sabouraud dextrose agar were used for bacteria and fungi, respectively. In the screening test for antimicrobial activity, $0.4 \%$ stock solutions were prepared by dissolving $40 \mathrm{mg}$ of the test compound in $10 \mathrm{ml}$ of distilled water or ethanol. The stock solutions were diluted in an orderly manner by successive piping of the solution in water containing nutrient agar or sabouraud dextrose agar to obtain 400, 200, 100, 50, 25, 10, 5, 2.5 and $1 \mathrm{ppm}$ concentrations of the compound. After sterilization of the agar, the solutions were poured into sterile Petri dishes, allowed to harden, and were then individually inoculated with one drop of each suspension, each containing a separate test microorganism. The inoculated dishes were then inoculated at $37^{\circ} \mathrm{C}$ for two days with bacteria strains and $25^{\circ} \mathrm{C}$ for five days with fungal strains, and examined for the presence or absence of 
Table 3.

Surface properties of amphoteric compounds

\begin{tabular}{|c|c|c|c|c|c|c|c|c|c|}
\hline \multirow{2}{*}{ Compd } & \multirow{2}{*}{$\begin{array}{c}\text { Surface } \\
\text { Tension } \\
\text { (dyne/cm) } \\
0.1 \%\end{array}$} & \multirow{2}{*}{$\begin{array}{c}\text { Interfacial } \\
\text { Tension } \\
\text { (dyne/cm) } \\
0.1 \%\end{array}$} & \multirow{2}{*}{$\begin{array}{c}\text { Kraft } \\
\text { Point } \\
{ }^{\circ} \mathrm{C} \\
1 \%\end{array}$} & \multirow{2}{*}{$\begin{array}{c}\text { Wetting } \\
\text { time } \\
(\mathrm{sec} .) \\
1 \%\end{array}$} & \multirow{2}{*}{$\begin{array}{c}\text { Emulsion } \\
\text { stability } \\
\text { (min.sec) }\end{array}$} & \multicolumn{2}{|c|}{$\begin{array}{c}\text { Foam power } \\
(\mathrm{mm}) 1 \% \\
\end{array}$} & \multirow{2}{*}{$\begin{array}{c}\mathrm{Ca}^{++} \text {stability } \\
\text { (ppm) }\end{array}$} & \multirow{2}{*}{$\underset{\text { mole } / \mathrm{I}}{\mathrm{Cmcx} 10^{-3}}$} \\
\hline & & & & & & Intial & $\begin{array}{l}\text { After } \\
5 \text { min }\end{array}$ & & \\
\hline $3 a$ & 31 & 7.5 & 17 & 65 & 260: 40 & 200 & 190 & 450 & 3.9 \\
\hline $3 b$ & 32 & 8.0 & 20 & 90 & 300: 30 & 220 & 205 & 380 & 3.7 \\
\hline $3 c$ & 36 & 8.5 & 25 & 123 & 350: 50 & 225 & 210 & 350 & 3.4 \\
\hline $4 a$ & 33 & 8.2 & 16 & 90 & 280: 55 & 155 & 149 & 1460 & 3.8 \\
\hline $4 b$ & 35 & 8.7 & 19 & 115 & $320: 25$ & 170 & 158 & 1350 & 3.6 \\
\hline $4 c$ & 37 & 9.0 & 23 & 132 & $360: 20$ & 190 & 173 & 1200 & 3.3 \\
\hline $5 a$ & 34 & 9.5 & 26 & 80 & 250: 10 & 180 & 165 & 1240 & 3.6 \\
\hline $5 b$ & 37 & 10.3 & 27 & 110 & 290: 25 & 200 & 189 & 1150 & 3.4 \\
\hline $5 c$ & 39 & 11.0 & 35 & 135 & $340: 22$ & 220 & 205 & 900 & 3.2 \\
\hline $6 a$ & 33 & 11.4 & 42 & 100 & 286: 40 & 185 & 163 & 1230 & 4.3 \\
\hline $6 b$ & 35 & 12.0 & 43 & 115 & 305: 55 & 200 & 184 & 1260 & 4.2 \\
\hline $6 c$ & 37 & 12.5 & 48 & 126 & 330: 30 & 230 & 210 & 850 & 3.5 \\
\hline $7 a$ & 31 & 6.7 & 48 & 85 & $254: 45$ & 148 & 155 & 560 & 6.1 \\
\hline $7 b$ & 33 & 7.0 & 50 & 100 & 280: 26 & 165 & 176 & 500 & 5.6 \\
\hline $7 c$ & 35 & 7.5 & 55 & 120 & 320: 10 & 188 & 185 & 450 & 4.6 \\
\hline $8 a$ & 32 & 8.7 & 17 & 36 & $227: 21$ & 145 & 164 & 1530 & 4.9 \\
\hline $8 b$ & 34 & 9.2 & 22 & 42 & 260: 39 & 159 & 179 & 1420 & 4.7 \\
\hline $8 c$ & 36 & 10.0 & 22 & 48 & 300: 38 & 183 & 196 & 1300 & 4.1 \\
\hline $9 a$ & 30 & 8.5 & 15 & 30 & $230: 47$ & 210 & 190 & 1260 & 4.1 \\
\hline $9 b$ & 32 & 9.0 & 16 & 31 & 270: 50 & 220 & 205 & 1150 & 3.8 \\
\hline $9 c$ & 34 & 9.5 & 20 & 36 & 310: 11 & 235 & 214 & 950 & 3.7 \\
\hline $10 a$ & 33 & 7.0 & 13 & 32 & 222: 50 & 190 & 174 & 1360 & 3.9 \\
\hline $10 b$ & 36 & 7.5 & 19 & 35 & 265: 11 & 200 & 183 & 1240 & 3.6 \\
\hline $10 c$ & 38 & 8.0 & 22 & 35 & 300: 38 & 205 & 191 & 1120 & 3.3 \\
\hline $11 a$ & 28 & 8.6 & 16 & 35 & 226: 17 & 180 & 166 & 1420 & 3.8 \\
\hline $11 b$ & 30 & 9.0 & 20 & 40 & 263: 33 & 200 & 182 & 1360 & 3.5 \\
\hline $11 \mathrm{c}$ & 32 & 9.4 & 23 & 45 & 290: 22 & 210 & 190 & 1300 & 3.1 \\
\hline $12 a$ & 32 & 8.7 & 19 & 39 & $227: 21$ & 155 & 168 & 1530 & 5.7 \\
\hline $12 b$ & 34 & 9.2 & 22 & 45 & 260: 39 & 160 & 172 & 1420 & 5.2 \\
\hline $12 c$ & 36 & 10.0 & 25 & 48 & 300: 38 & 165 & 206 & 1300 & 4.6 \\
\hline
\end{tabular}

Error of measurements was:

Surface and interfacial tensions $= \pm 0.1 \mathrm{dynes} / \mathrm{cm}$.

Kraft point $= \pm 1^{\circ} \mathrm{C}$

Koam hoight $= \pm 1^{\circ} \mathrm{C}$

Foam height $= \pm 2 \mathrm{~mm}$

Wetting time $= \pm 1 \mathrm{sec}$
Emulsion stability $= \pm 1 \mathrm{~min}$

growth. Antimicrobial activities are represented in terms of minimum inhibitory concentrations (MIC).

\section{RESULTS AND DISCUSSION}

A number of amphoteric surfactants was synthesized by the reaction of alkyl ester epoxides and amino acids (glycine, alanine, valine, isoleucine, phenylalanine, tyrosine, serine, threonine, aspartic and anthranilic acid). This group can be prepared from readily accessible starting materials without expensive reagents or special equipment. In general, the synthetic procedures gave relatively high yields in two simple synthetic steps.

\subsection{Surface active properties}

The surface properties (surface and interfacial tension, Kraft point, wetting power, foaming properties, emulsifying power and critical micelle 
concentration) of well purified compounds were investigated in distilled water. These surfactants show relatively high surface activity and a comparative study between the structure and the result was made.

\subsubsection{Surface and interfacial tension}

The measurements of the individual compounds are listed in (Table 3). The results indicated that, a linear relationship was observed between surface and interfacial tension and alkyl chain length (as the number of carbon atoms in the alkyl chain increases the surface and interfacial tension increases).

\subsubsection{Kraft point}

The Kraft point of a surfactant molecule is the temperature at which $1 \%$ dispersion solution becomes clear under gradual heating. The Kraft points of all synthesized amphoteric surfactants are also summarized in (Table 3). Although the Kraft points increase in the order $\mathrm{C}_{18}>\mathrm{C}_{12}>\mathrm{C}_{8}$ no remarkable difference among three homologues was observed. However, as the molecular weight of an amino acid increases the Kraft point increases.

\subsubsection{Wetting power}

The wetting time of the tested amphoteric surface active agents was determined by calculating the

Table 4.

Biodegradability of Amphoteric Products

\begin{tabular}{|c|c|c|c|c|c|c|c|}
\hline Compds. & 1st day & 2nd day & 3rd day & 4th day & 5th day & 6th day & 7th day \\
\hline $3 a$ & 44 & 52 & 60 & 71 & 84 & - & - \\
\hline $3 b$ & 41 & 49 & 45 & 65 & 77 & 92 & - \\
\hline $3 c$ & 38 & 45 & 51 & 62 & 70 & 84 & - \\
\hline $4 a$ & 45 & 55 & 62 & 69 & 78 & 88 & 97 \\
\hline $4 b$ & 41 & 50 & 58 & 66 & 75 & 85 & - \\
\hline $4 c$ & 37 & 45 & 54 & 63 & 71 & 83 & 91 \\
\hline $5 a$ & 41 & 51 & 64 & 75 & 90 & - & - \\
\hline $5 b$ & 38 & 46 & 58 & 72 & 87 & 93 & - \\
\hline $5 c$ & 35 & 42 & 53 & 67 & 82 & 90 & - \\
\hline $6 a$ & 43 & 50 & 63 & 75 & 92 & - & - \\
\hline $6 b$ & 39 & 47 & 58 & 71 & 88 & 93 & - \\
\hline $6 c$ & 37 & 44 & 54 & 68 & 75 & 89 & 94 \\
\hline $7 a$ & 40 & 49 & 58 & 69 & 82 & 98 & 95 \\
\hline $7 b$ & 37 & 46 & 55 & 64 & 79 & 84 & 92 \\
\hline $7 c$ & 35 & 42 & 51 & 60 & 74 & 86 & 92 \\
\hline $8 a$ & 41 & 55 & 66 & 77 & 89 & - & - \\
\hline $8 b$ & 39 & 53 & 62 & 72 & 86 & 93 & - \\
\hline $8 c$ & 33 & 50 & 58 & 68 & 83 & 89 & 95 \\
\hline $9 a$ & 49 & 56 & 67 & 81 & 90 & - & - \\
\hline $9 b$ & 42 & 52 & 64 & 75 & 87 & 94 & - \\
\hline $9 c$ & 39 & 47 & 59 & 69 & 79 & 89 & 96 \\
\hline $10 a$ & 48 & 58 & 69 & 80 & 93 & - & - \\
\hline $10 b$ & 45 & 54 & 65 & 77 & 89 & 94 & - \\
\hline $10 c$ & 41 & 51 & 61 & 73 & 84 & 90 & - \\
\hline $11 a$ & 50 & 53 & 64 & 76 & 89 & - & - \\
\hline $11 b$ & 44 & 50 & 60 & 71 & 86 & 92 & - \\
\hline $11 \mathrm{c}$ & 38 & 46 & 55 & 66 & 79 & 89 & 97 \\
\hline $12 a$ & 47 & 55 & 66 & 77 & 81 & 89 & 99 \\
\hline $12 b$ & 43 & 53 & 62 & 72 & 79 & 86 & 93 \\
\hline $12 c$ & 39 & 50 & 58 & 68 & 72 & 83 & 90 \\
\hline
\end{tabular}

Error of calculations was: Biodegradation rate $= \pm 0.5 \%$. 
sinking time in seconds of a grey cotton cloth in the surfactant solution. The synthesized surfactants showed good performance for wetting power (shorter sinking time). Compounds (8a, 9a-c, 10a-c and 11a) recorded excellent wetting power which makes them useful for extensive applications in the textile industry.

\subsubsection{Foaming power}

The foaming properties of all the synthesized surfactants were measured by the Ross and Miles method (Ross et al., 1941). Amphoteric surfactants showed good foam production as well as better foam stability above the (CMC). On the other hand, amphoteric surfactants containing an aromatic ring such as (7a-c, 8a-c and 12a-c) showed poor foaming properties. Extremely low foaming can probably be ascribed to the low solubility (low hydrophilicity) of the compounds in water. Table 3 shows the foam production and foam stability of all the synthesized amphoteric surfactants.

\subsubsection{Emulsifying power}

Emulsification is one of the most important properties of surfactants. In many textile processes such as scouring and dyeing, it is necessary to introduce surfactants into a bath to remove oily impurities from the fibers. On the other hand, amphoteric surfactants with good emulsion stability have been used in such fields as shampoos and cosmetics, emulsion paints and in the textile industry. The emulsification power is determined and listed in (Table 3 ). The results reflect the fact that as the alkyl chain length increases the emulsifying power increases.

\subsection{6. $\mathrm{Ca}^{++}$- Stability}

The calcium ion stability results of amphoteric surfactants are shown in (Table 3). High calcium stability values show that the prepared surfactants can be used in hard water. The calcium stability

Table 5.

Antimicrobial activity of amphoteric compounds.

\begin{tabular}{|c|c|c|c|c|c|c|c|c|c|c|c|c|}
\hline Compds & $\begin{array}{l}\text { Stophylococcus } \\
\text { Aureus } \\
\text { MIC }\end{array}$ & $\begin{array}{c}\text { Bacillus } \\
\text { Subtiles } \\
\text { MIC }\end{array}$ & $\begin{array}{l}\text { Sarcina } \\
\text { Lutea } \\
\text { MIC }\end{array}$ & $\begin{array}{l}\text { Escherichia } \\
\text { Coli } \\
\text { MIC }\end{array}$ & $\begin{array}{l}\text { Salmonella } \\
\text { Trphi } \\
\text { MIC }\end{array}$ & $\begin{array}{c}\text { Pseudomonas } \\
\text { Aeruginosa } \\
\text { MIC }\end{array}$ & $\begin{array}{c}\text { Candida } \\
\text { Albicans } \\
\text { MIC }\end{array}$ & $\begin{array}{c}\text { Saccharomyces } \\
\text { Cerevisiae } \\
\text { MIC }\end{array}$ & $\begin{array}{c}\text { Alternaria } \\
\text { Humicala } \\
\text { MIC }\end{array}$ & $\begin{array}{l}\text { Fusazium } \\
\text { Oxysporum } \\
\text { MIC }\end{array}$ & $\begin{array}{l}\text { Asperdillus } \\
\text { Flavus } \\
\text { MIC }\end{array}$ & $\begin{array}{l}\text { Microsporium } \\
\text { Gypseum } \\
\text { MIC }\end{array}$ \\
\hline $3 a$ & $>400$ & 100 & 50 & 10 & 200 & 50 & 25 & $>400$ & 2.5 & 100 & 200 & 400 \\
\hline $3 b$ & 200 & 25 & 50 & 10 & 400 & 100 & 10 & 200 & 10 & 100 & 100 & 400 \\
\hline $3 c$ & 100 & 50 & 100 & 5 & 100 & 100 & 10 & 200 & 10 & 50 & 50 & $>400$ \\
\hline $4 a$ & 400 & 25 & 50 & 10 & 200 & 50 & 25 & 100 & 2.5 & 100 & 100 & 400 \\
\hline $4 b$ & $>400$ & 25 & 50 & 10 & 400 & 100 & 10 & 50 & 10 & 100 & 25 & $>400$ \\
\hline $4 c$ & 200 & 50 & 25 & 5 & 100 & 100 & 10 & 50 & 2.5 & 50 & 50 & 200 \\
\hline $5 a$ & 100 & 50 & 200 & 10 & 200 & 50 & 2.5 & 100 & 2.5 & 50 & 50 & 200 \\
\hline $5 b$ & 50 & 25 & 50 & 10 & 200 & 100 & 1 & 50 & 2.5 & 25 & 25 & 200 \\
\hline $5 c$ & 25 & 50 & 100 & 5 & 100 & 100 & 2.5 & 100 & 2.5 & 50 & 50 & 50 \\
\hline $6 a$ & 100 & 100 & 200 & 10 & 50 & 50 & 25 & 100 & 2.5 & 100 & 100 & 200 \\
\hline $6 b$ & 25 & 25 & 50 & 10 & 50 & 100 & 10 & 50 & 2.5 & 50 & 50 & 200 \\
\hline $6 c$ & 10 & 50 & 100 & 5 & 25 & 100 & 10 & 100 & 1 & 50 & 50 & 50 \\
\hline $7 a$ & 10 & 25 & 50 & 10 & 50 & 50 & 25 & 100 & 10 & 50 & 100 & 200 \\
\hline $7 b$ & 1 & 10 & 50 & 10 & 25 & 100 & 10 & 10 & 2.5 & 25 & 50 & 100 \\
\hline $7 c$ & 2.5 & 2.5 & 25 & 5 & 10 & 100 & 1 & 2.5 & 2.5 & 10 & 50 & 50 \\
\hline $8 a$ & 100 & 100 & 200 & 10 & 200 & 50 & 25 & 200 & 2.5 & 100 & 200 & 50 \\
\hline $8 b$ & 50 & 25 & 50 & 10 & 400 & 100 & 10 & 200 & 2.5 & 100 & 100 & 200 \\
\hline $8 c$ & 25 & 50 & 100 & 5 & 100 & 100 & 10 & 100 & 2.5 & 50 & 50 & 50 \\
\hline $9 a$ & $>400$ & 100 & 200 & 10 & 200 & 50 & 25 & $>400$ & 10 & 100 & 200 & 200 \\
\hline $9 b$ & 200 & 25 & 50 & 10 & 400 & 100 & 10 & 200 & 2.5 & 100 & 100 & 200 \\
\hline $9 c$ & 200 & 50 & 100 & 5 & 100 & 100 & 10 & 100 & 2.5 & 50 & 50 & $>400$ \\
\hline $10 \mathrm{a}$ & 50 & 100 & 200 & 10 & 50 & 50 & 25 & $>400$ & 50 & 100 & 200 & 200 \\
\hline $10 \mathrm{~b}$ & 50 & 25 & 50 & 10 & 50 & 100 & 10 & 400 & 10 & 100 & 100 & 50 \\
\hline $10 \mathrm{c}$ & 25 & 50 & 100 & 5 & 25 & 100 & 10 & 400 & 10 & 50 & 50 & 50 \\
\hline $11 a$ & 50 & 100 & 200 & 10 & 200 & 50 & 25 & 50 & 100 & 100 & 200 & 400 \\
\hline $11 b$ & 50 & 25 & 50 & 10 & 400 & 100 & 10 & 10 & 50 & 100 & 100 & $>400$ \\
\hline $11 c$ & 10 & 50 & 100 & 5 & 100 & 100 & 10 & 2.5 & 50 & 50 & 50 & 400 \\
\hline $12 a$ & $>400$ & 100 & 200 & 10 & 200 & 50 & 25 & 10 & 2.5 & 100 & 200 & 50 \\
\hline $12 b$ & 400 & 25 & 50 & 10 & 400 & 100 & 10 & 2.5 & 2.5 & 100 & 100 & 200 \\
\hline $12 \mathrm{c}$ & 100 & 50 & 100 & 5 & 100 & 100 & 10 & 2.5 & 2.5 & 50 & 50 & 50 \\
\hline
\end{tabular}

Origin of cultures: Botany Department, Faculty of Science, Benha University, Egypt. 
decreased with the increase in the molecular weight of the hydrophobic part of the surfactant under the conditions of constant temperature.

\subsubsection{Critical micelle concentration (CMC)}

The critical micelle concentration values of the prepared amphoteric surfactants were determined using the electrical conductivity method. The results showed that as the hydrophobic part increases the $\mathrm{CMC}$ values decrease, this means that aliphatic compounds exhibit larger intermolecular hydrophilic interactions, making it easier for them to form aggregates in water than those which contain an aromatic ring. Also, the results of CMC measurements reflect the fact that as the length of alkyl chain increases the CMC decreases.

\subsection{Biodegradability}

The biodegradability of the tested compounds after one week was determined and listed in (Table 4). Each experiment was repeated three times, and the results are reported as averages of three values. For example, compound (9a) was $100 \%$ degraded in 6 days and $81 \%$ degraded in 4 days which makes it an excellent biodegradable surface active agent.

\subsection{Antimicrobial activity}

All the synthesized surface active agents were screened for antimicrobial activity against Grampositive and Gram-negative bacterial strains and fungal strains. The minimum inhibitory concentrations (MIC) for the compounds tested are given in (Table 5). Compounds (5a,b, 6b,c and 7a,b,c) showed a broad spectrum of antimicrobial activity. On the other hand compounds (3c, 4b, 9c, and 11b,c) showed no significant antimicrobial activities.

\section{BIBLIOGRAPHY}

Amin. M. S., Eissa. A. M. F, Shaaban. A. F., El-Sawy. A. A. and El-Sayed. R. 2004 "New heterocycles having a double characters as antimicrobial and surface active agents.Part 1: Nonionic compounds from fatty acid isothiocyanate". Olaj Szappan, Kozmetika 53,124-128.

Andersen. D. L. 1957 J. Am. Oil. Chem. Soc. 34, 188-190.

Bluestein. C., Rosenblatt. W., Clark. J and Stefcik. A. (1973) J. Am. Oil. Chem. Soc. 50, 532-535.

Bristiline R. G., Maurer. E. W., Smith. F. D and Linfield. W. M. (1980) J. Am. Oil. Chem. Soc. 75, 98-102.

Christophe. D., Fatima. A., Karine. V., Guita. E. and Isabelle. R. (2002) Longmuir. 18, 10168-10175.
Eissa. A. M. F. (2002) "Anionic surface active agents from fatty acid hydrazides containing heterocyclic moiety". J. Olaj Szappan Kozmetika, 51, 155-161.

Eissa. A. M. F and Osman. F. (1996) "Cationic surface active agents". Bull. N R C. Egypt. 21 (3), 245-252.

Eissa. A. M. F. (1995) "Amphoteric surface active agents". Grasas Y Aceites, 46 (4-5), 240-4.

Eissa. A. M. F and Ahmed. M. H. M. (2003) "Nonionic surface active agents containing heterocyclic moiety". Olaj Szappan Kozmetika 52, 11-17.

El-Dougdoug. W. I. A., Hebash. K. A. H and Eissa. A. M. F. (2001) "Cationic surfactants from local magnifera indica fat". J. Olaj Szappan Kozmetika. 50 (4) 143148

El-Sawy. A. A., Essawy. S. A., El-Sukkary. M. M and Eissa. A. M. F. (1992) "Surfactants from 2-hydroxy fatty acids I. Sulphonation products of 2-hydroxy fatty acids, esters, diols and alkanolamines". J. Serb. Chem. Soc. 56, 25-28.

El-Sukkary. M. A., El-Sawy. A. A and El-Dib. F. (1987) "Synthetic Detergents from Crude rice bran oil". Hungarian Journal of Industrial Chemistry 15, 317320.

Eter, E. T., Richard. R. E. and Darid. A. (1974) "Biodegradable surfactants derived from corn starch". J. Am. Oil Chem. Soc. 51, 486 - 494.

Findly. A. (1963) "Practical physical chemistry". $6^{\text {th }}$ Ed. Longmans, London. 1040.

Hikota. T and Moerugo. K. (1979). J. Am. Oil. Chem. Soc. 56 537-541.

Herrmann. K. W. (1962). J. Phys. Chem. 66, 295-298.

Hironari. F. and Kimihiro. M. (2002) Jpn. Kokai Tokkyo Koho. 26, 9-13.

Klrvens. H. B. (1953). J. Am. Oil. Chem. Soc. 30, 74-76.

Masuyama. A., Akiyama. K and Okahara. M. (1987) "Surface active hydroxamic acid. II. Comparison of surface properties of hydroxamic acids with ketones and methyl esters with similar hydrophilic and lipophilic structure". J. Am. Oil. Chem. Soc. 64, 10401043.

Infante. M. R., Perez. L., Pinazo. A., Clapes. P and Moran. C. (2003). Surfactant Science Series: Novel surfactants. 114, 193-215

Marion. H., Deisenroth. T and Jemnings. J. (2002). Mueller, Karl Friedrich. 15, 42-47.

Nasreddine. M., Szonyi. S and Cambon. A. (1993) J. Am. Oil. Chem. Soc. 70 (1),105-107.

Somaya. A. R, Eissa. A. M. F, Nadia. A and Ahmad. M. N. (1998) "Synthesis and characterization of some peptides having surface activity using polyethylene glycol". J. Pharm. Sci. 7, 27-32.

Ross. J and Miles. G. (1941) J. Oil and Soap. 18, 99-102.

Takeshi. H. (1970) Bull. Chem. Soc, 43, 2236-2239.

Tokiwa, F and Ohki. K. (1967) J. Phys. Chem. 71, 18241827.

Wiel. J. K., Smith. F. D., Stirton. A. J. and Bistine. R. G. (1963). "Long chain alkane-sulphonates and 1 . hydroxy-2-alkanesulphonates: Structure and property relations" J. Am. Oil. Chem. Soc. 40, 538-541.

Recibido: Mayo 2005 Aceptado: Marzo 2006 